\title{
Avances en el estudio cinético de la transesterificación de aceite crudo de algodón.
}

\section{Advances in the kinetic study of the transesterification of raw cottonseed oil.}

Presentación: 18/10/2019

\section{Doctorando:}

\section{Alfredo Fabian Sequeira}

Centro UTN de Investigación QUIMOBI (Química Orgánica Biológica), Facultad Regional Resistencia, Universidad Tecnológica Nacional

alfreseq@gmail.com

\section{Directora:}

\section{Dra. Ester Chamorro}

\section{Codirector:}

\section{Dr. Gustavo Aucar}

\section{Resumen}

En el marco de la Tesis Doctoral denominada "Modelado computacional de la transesterificación de lípidos saponificables presentes en el aceite de semillas de algodón con catálisis homogénea" se presentan los datos relativos a la determinación del camino de reacción que tiene lugar en la transesterificación del aceite crudo de algodón utilizando herramientas computacionales.

Palabras claves: Aceite crudo de algodón, Transesterificación, Cálculos Computacionales.

\begin{abstract}
Within the develop of the Doctoral Thesis "Computational modeling of the transesterification of saponifiable lipids present in cottonseed oil with homogeneous catalysis", in this document are presented data regarding the determination of the reaction path that takes place in the transesterification of cottonseed raw oil using computational calculations.
\end{abstract}

Keywords: Cottonseed raw oil, Transesterification, Computational calculations, etc.

\section{Introducción}

La sustitución de los combustibles denominados fósiles o tradicionales, derivados del petróleo, por otros de origen vegetal o animal, denominados biocombustibles cobra, en nuestros días, cada vez mayor importancia económica y medioambiental, dado que los biocombustibles provienen de una fuente renovable $[1,2,3,4,5,6,7,8$, $9,10,11]$.

Estos biocombustibles constituyen una realidad cotidiana para un sin número de productores radicados en la Argentina, especialmente en aquellas áreas Agroindustriales que sufren la falta de combustible fósiles, localizadas, particularmente, en la región Nordeste del país [4, 8, 10, 11, 12, 13].

El biodiesel se define como un biocombustible oxigenado que se obtiene a partir de grasas animales y de distintos tipos de aceites vegetales saponificables. Entre los aceites vegetales se destacan, como materia prima más usual para 
la obtención de biodiesel, los de: girasol, algodón, soja e incluso los aceites usados provenientes de la industria alimentaria.

En la actualidad es relevante explorar la posibilidad de obtener biodiesel a partir de fuentes que no compitan con aquellas destinadas a la producción de alimentos, estos biocombustibles son denominados en general de segunda y tercera generación. En este marco y dado que en la región del Nordeste Argentino el algodón está destinado principalmente a la extracción de fibra vegetal por lo que sus semillas oleaginosas constituyen un residuo de la industria y que en general para la eliminación de las mismas se recurre a la incineración al aire libre, el aceite crudo de semilla de algodón resulta ser una materia prima ideal en la obtención de biodiesel $[8,10,12,13,14,15,16,17]$.

Desde el año 2005 se han desarrollado en el seno del Centro UTN de Investigación QUIMOBI (Química Orgánica Biológica), tareas tendientes a mejorar los procesos de obtención de biodiesel a partir de aceites vegetales crudos que presentan elevada acidez, en particular el proveniente de semilla de algodón $[4,5,8,10,12,13,14,15,19,20]$, al tiempo resulta relevante destacar que desde el año 2002 se han desarrollado tareas relativas al cálculo computacional abordándose el tema de la relación estructura-actividad de feromonas de insectos plaga y el estudio teórico estático y dinámico de biomoléculas [21, 22, 23] adquiriéndose mediante la ejecución de las mencionadas tareas de investigación los conocimientos críticos necesarios para abordar el modelado computacional de la reacción de transesterificación de aceite crudo de semilla de algodón.

En el ámbito internacional, en lo que hace al modelado computacional de la reacción de transesterificación, solo se aborda el estudio de estructuras de aceites saponificables a las que podría denominarse, en el mejor de los casos, como ideales ya que no coinciden con la composición real de ningún aceite vegetal existente [24, 25].

Con la ejecución del trabajo de la Tesis Doctoral denominada "Modelado computacional de la transesterificación de lípidos saponificables presentes en el aceite de semillas de algodón con catálisis homogénea" se pretende, como Objetivo General, formular la cinética de la reacción de transesterificación de lípidos saponificables presentes en el aceite de semillas de algodón con catálisis homogénea, mediante herramientas del área de modelado computacional, para contribuir con el conocimiento en la producción de biodiesel.

En el presente informe de avances se detallan los resultados obtenidos a partir de cálculos computacionales destinados a lograr la comprensión de la reacción de transesterificación.

\section{Resultados}

Se ha desarrollado el Modelado Matemático de la cinética de la reacción de transesterificación y la prospección probabilística de la composición de lípidos saponificables presentes en aceites vegetales con un particular interés en los resultados obtenidos para el aceite crudo de algodón [26].

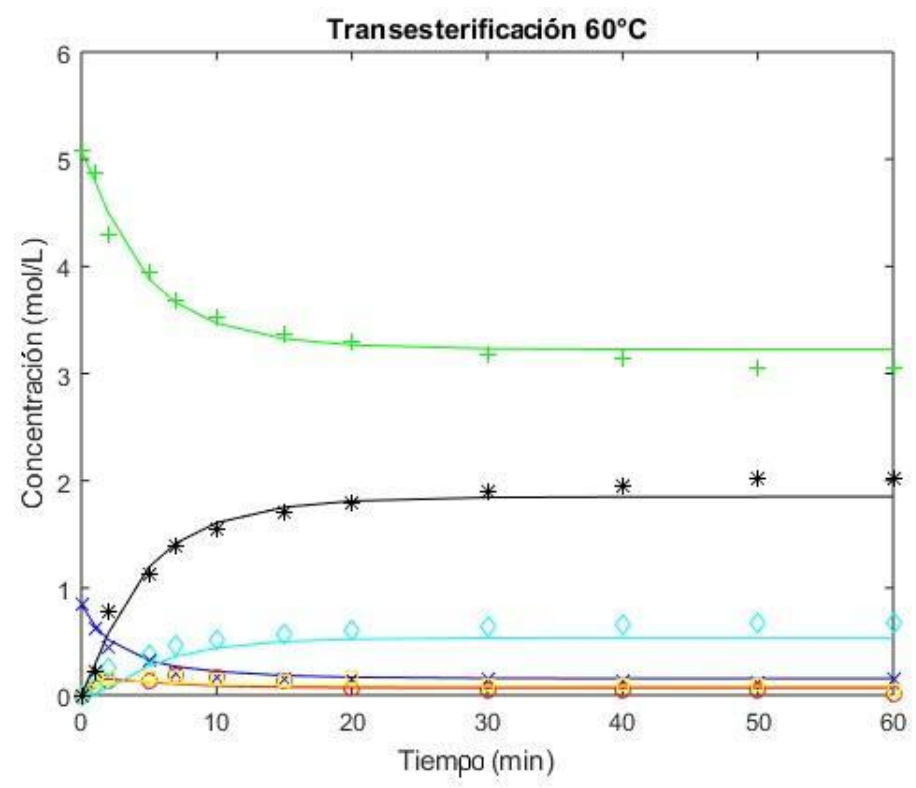

Puede observarse en la Figura 1 la variación de la concentración, a lo largo del tiempo, de las especies involucradas de la transesterificación a $60^{\circ} \mathrm{C}$ del Aceite de Algodón, los valores experimentales se representan mediante marcas, mientras que las calculadas por el software se representan en líneas continuas.

Figura 1. Variación a lo largo del tiempo de la composición de la mezcla reaccionante durante la transesterificación a $60^{\circ} \mathrm{C}$ de Aceite de Algodón (Concentraciones: Triglicéridos - $x$ - azul, Diglicéridos " $O$ " rojo, Monoglicéridos " $O$ " amarillo, Glicerol " $\nabla$ " celeste, FAME "*” negro y Metanol "+" verde).

Los valores de $\mathrm{R}^{2}$ correspondientes al ajuste entre las curvas experimentales y calculadas permiten concluir que se han establecido correctamente los lineamientos básicos para el Modelado Matemático de la Cinética de

la Reacción de Transesterificación utilizando el Software Matlab $\left(\mathrm{R}^{2}>0,8\right)$ para triglicéridos, FAME, glicerol y metanol, mientras que para monoglicéridos y diglicéridos se encuentran alejados del $\mathrm{R}^{2}$ óptimo ya que se trata de 
productos intermedios que se generan y se consumen a lo largo de la reacción y que por lo tanto juegan un rol no decisivo en la misma.

Utilizando datos propios y datos obtenidos a partir de publicaciones científicas internacionales se ha detectado que la composición estadística de los lípidos saponificables presentes en los aceites de girasol, soja y algodón contienen a los ácidos grasos Palmitico (P), Oleico (O) y Linoleico (L). Aplicando la ecuación de Probabilidad de Combinación $\left(\mathrm{PB}=\mathrm{Pi}^{*} \mathrm{P}+\mathrm{Pi}^{*} \mathrm{O}+\mathrm{Pi}{ }^{*} \mathrm{~L}\right)$ donde $\mathrm{Pi}$ es el Número de Orden, $\mathrm{P}$ es el porcentaje de Palmítico en la mezcla, $\mathrm{O}$ es el porcentaje de Oleico en la mezcla y $\mathrm{L}$ es el porcentaje de Linoleico en la mezcla se ha determinado que para el aceite de algodón las combinaciones más relevantes son: L-L-L (9.59\%), P-L-L (7.83\%), L-P-L (7.83\%), 0-L-L (7,31\%) y L-O-L (7,31\%).

Con los valores de la Probabilidad de la Combinación (PB), correspondientes a los Lípidos Saponificables presentes en el Aceite de Algodón, Soja y Girasol, es posible decidir de manera correcta cómo construir las estructuras de los triglicéridos cuya Probabilidad de Combinación resulta ser la más relevante a fin de simular la reacción de Transesterificación mediante la aplicación de un Software de Química Computacional como por ejemplo el Gaussian 16W.

A partir de los resultados obtenidos para determinar las combinaciones más relevantes se construyeron los modelos moleculares correspondientes a los triglicéridos saponificables de mayor relevancia probabilística presentes en el aceite de algodón, se han desarrollados los cálculos computacionales necesarios para detectar las conformaciones de mínima energía en cada uno de los 5 triglicéridos estadísticamente más relevantes, recurriendo al Software Gaussian 09 utilizando la Teoría del Funcional de la Densidad (DFT) a nivel B3LYP/6-31G(d), se han desarrollado los Barridos (Scan) de los Diedros D1 (Figura 2.) y D2 (Figura 3.) correspondientes a la cadena de glicerol del triglicérido. presentes en el aceite de algodón [Publicación en Elaboración].

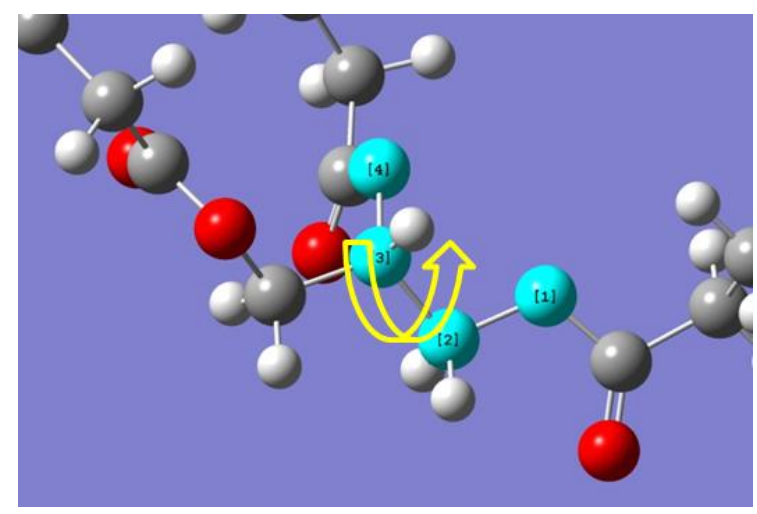

Figura 2. Angulo Diedro D1.

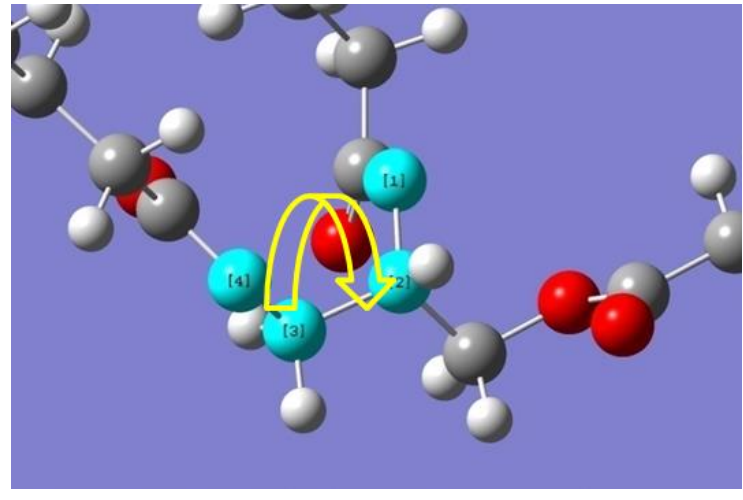

Figura 3. Angulo Diedro D2.

De los cálculos realizados se ha detectado que la estructura de menor energía resulta ser la que corresponde al triglicérido formado por la combinación de los ácidos grasos linoleico-oleico-linoleico (que cuenta con una relevancia estadística de 7,31\% en el aceite de algodón) con un valor de $-1684024,53 \mathrm{kcal} / \mathrm{mol}$ y con ángulos diedros que corresponden a las posiciones gauche $\left(277,243^{\circ}\right)$ para D1 y anti $\left(170,683^{\circ}\right)$ para D2. Estos valores de ángulo diedro otorgan a la estructura una conformación a la que es posible calificar como "distendida".

En la Tabla 1. puede observarse el resultado de los barridos (scan) de los diedros D1 y D2 de cada una de las 5 estructuras, el valor de la energía expresado en kcal por mol y el salto de energía entre la estructura de valor mínimo y la correspondiente a cada estructura luego de la optimización final $(\Delta \mathrm{E})$.

Tabla 1. Valores correspondientes a las configuraciones de Mínima Energía de los Triglicéridos Saponificables de mayor relevancia probabilística presentes en el Aceite de Algodón

\begin{tabular}{ccccc}
\hline Combinación de Ácidos Grasos & Diedro D1 & Diedro D2 & E (kcal/mol) & $\Delta$ E \\
\hline Linoleico-Palmítico-Linoleico (L-P-L) & $294,021^{\circ}$ & $62,591^{\circ}$ o & $-1635457,33171$ & $-48567,20440$ \\
Palmítico-Linoleico-Linoleico (P-L-L) & $158,729^{\circ}$ & $291,009^{\circ}$ & $-1635458,46123$ & $-48566,07488$ \\
Linoleico-Linoleico-Linoleico (L-L-L) & $50,591^{\circ}$ & $287,899^{\circ}$ & $-1683249,81613$ & $-774,71998$ \\
Oleico-Linoleico-Linoleico (O-L-L) & $298,789^{\circ}$ & $163,560^{\circ}$ & $-1684019,79302$ & $-4,74309$
\end{tabular}


En la Tabla 1. también es posible observar que los saltos energéticos de mayor valor $(\Delta \mathrm{E}=-48567,20440)$ y por lo tanto más inestables contienen al ácido graso palmítico que resulta ser saturado (L-P-L) y presenta una cadena corta de solamente 16 átomos de carbono, mientras que el oleico es un ácido graso monoinsaturado con una cadena de 18 átomos de carbono que forma parten de la serie omega 9 y el linoleico es un ácido graso que presenta dos insaturaciones conjugadas con una cadena de 18 átomos de carbono que forma parten de la serie omega 6.

En las Figuras 4 a 8 es posible observar las estructuras finales de los 5 triglicéridos saponificables de mayor relevancia probabilística presentes en el aceite de algodón, en todos los casos se visualiza que las estructuras presentan una configuración "distendida", resulta interesante observar que el triglicérido optimizado de la combinación Linoleico-Oleico-Linoleico (Figura 8.) parece estar en un único plano molecular (similar a la palma de la mano abierta totalmente), además en las Tabla 3 puede verse también que este triglicérido es el que presenta la menor energía y por lo tanto la mayor estabilidad.

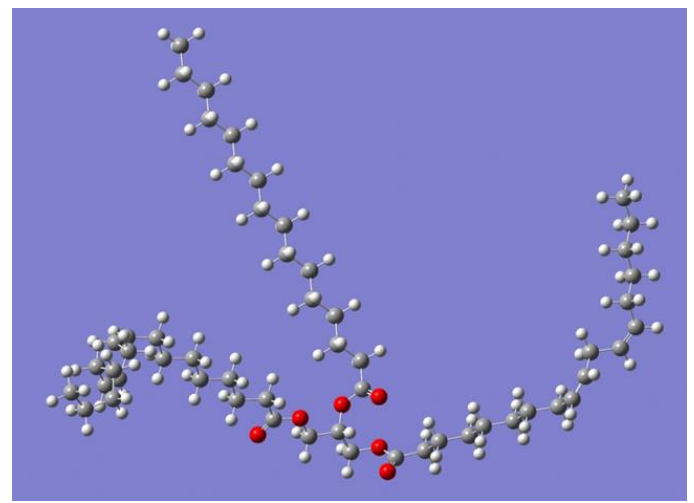

Figura 4. Triglicérido L-P-L optimizado.

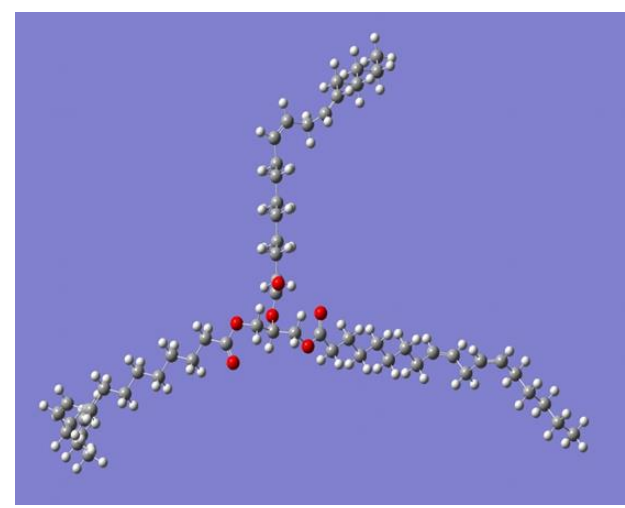

Figura 6. Triglicérido L-L-L optimizado.

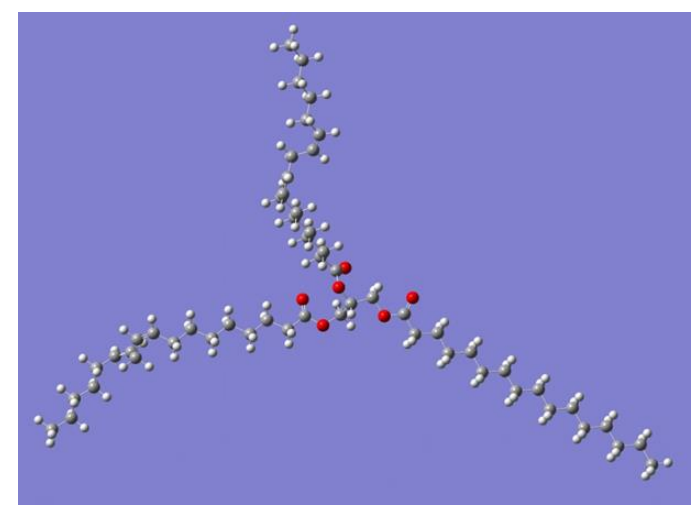

Figura 5. Triglicérido P-L-L optimizado.

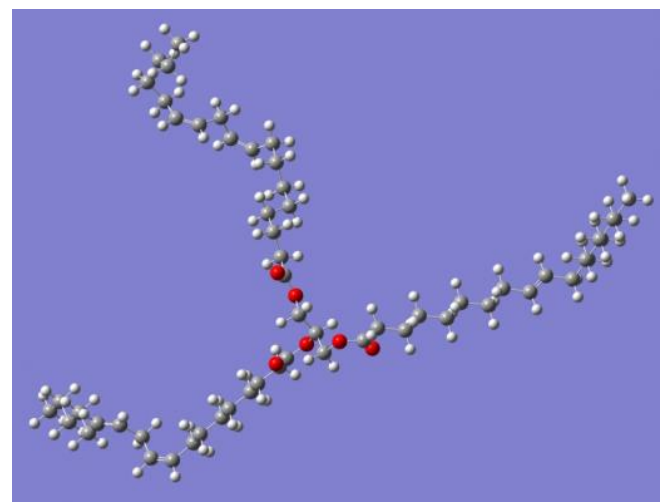

Figura 7. Triglicérido O-L-L optimizado.

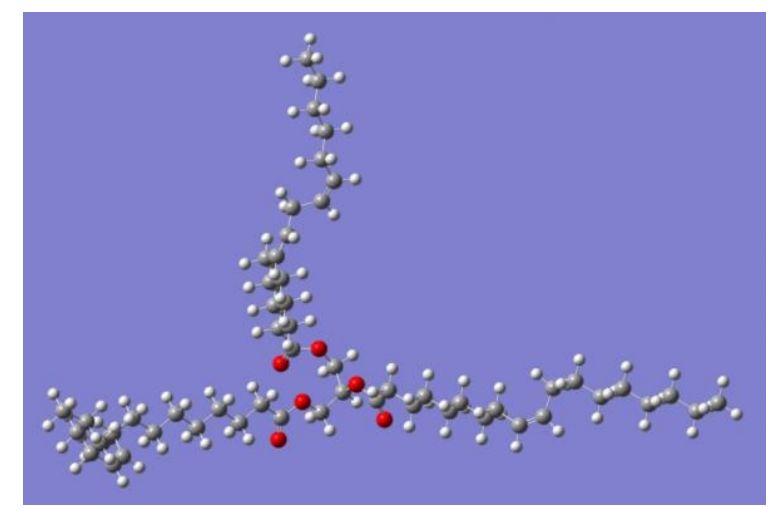

Figura 8. Triglicérido L-O-L optimizado. 


\section{Perspectiva}

A partir de los datos expuestos se plantea continuar con los cálculos computacionales a fin de establecer el camino de reacción que tiene lugar en la transesterificación del aceite crudo de algodón para ello será necesario localizar a los orbitales moleculares HOMO y LUMO de cada una de las estructuras y desagregarlas paulatinamente a fin de localizar a los orbitales moleculares mencionados en los digliceridos y monogliceridos derivados correspondientes, que como se observa en la Figura 1. si bien son intermediarios que se generan y se consumen a lo largo de la reacción deben tenerse en cuenta.

Para la cuantificación energética de la reacción de transesterificación deberá tenerse especial consideración con las configuraciones L-O-L de menor energía y L-P-L de mayor energía, ya que las restantes configuraciones probables arrojaran energías intermedias. Para concretar estos cálculos se utilizaran los antecedentes de cálculo descriptos y explicados en el libro "Exploring Chemistry with Electronic Structure Methods" que acompaña a los software Gaussian 16W y GaussView 6.0.

\section{Referencias}

1. Zhang Y.; Dubé M. A.; McLean D. D.; Kates M.; (2003). Biodiesel production from waste cooking oil: 1. Process design and technological assessment; Bioresourse Technology 89:1-16.

2. Zhang Y.; Dubé M. A.; McLean D. D.; Kates M.; (2003). Biodiesel production from waste cooking oil: 2. Economic assessment and sensitivity analysis. Bioresourse Technology 90:229-240.

3. Van Gerpen J.; (2005). Biodiesel processing and production. Fuel Processing Tecnology 86:1097-1107.

4. Sequeira A.; Chamorro E.; Morales W.G.; Dagnino P.; Cáceres M. y Diaz A.; (2007). Estudio experimental sobre la optimización de la conversión de aceite de algodón en biodiesel. AVERMA; Editorial INENCO; 11:01.43-01.46.

5. Morales W.G.; Perrault G.R.; Polich N.; Sequeira A.; Chamorro E.; Guiacopello N.; Soria S.; (2010). Comparación de biocombustibles y combustible diésel de origen fósil en motor diesel. I Jornadas Regionales de Investigación en Ingeniería. ISBN: 978-950-42-0130-4.

6. Ramos Abril J.; Cortés Gómez J.; Marriaga Cabrales N.; (2010). Biodiesel a partir de bioetanol y aceite de palma. Estudio tecnoeconómico. Dyna; 77(164):264-273. Medellín; Colombia.

7. Ashish Birla; Bhaskar Singh; Upadhyay S.N.; Sharma Y. C.; (2012). Kinetics studies of synthesis of biodiesel from waste frying oilusing a heterogeneous catalyst derived from snail shell. Bioresource Technology 106:95-100.

8. Morales W. G.; Sequeira A. F.; Chamorro E.; Braga M.; Sobral A.; De Sousa H. y Herrero E. R.; (2012). Catálisis heterogénea en la obtención de biodiesel. Avances en Energías Renovables y Medio Ambiente. $16(9): 15-22$

9. Rubio Caballero J. M.; Santamaría González J.; Mérida Robles J.; Moreno Tost R.; Alonso Castillo M. L.; Vereda Alonso E.; Jiménez López A.; Maireles Torres P.;(2013). Calcium zincate derived heterogeneous catalyst for biodiesel production by ethanolysis. Fuel 105:518-522.

10. Morales Walter G., Zurko Vanessa, Sequeira Alfredo, Chamorro Ester. (2014). Evaluación de variables para la producción de metil ésteres a partir de aceite de semilla de algodón asistido por ultrasonido. Extensionismo, Innovación y Transferencia Tecnológica, Claves para el desarrollo (FACENA) 1:119-124

11. Dagnino Eliana, Medina Carlos, Beligni María y Chamorro Ester. (2014). Evaluación de lípidos extraídos de microalgas nannochloropsis oculata para la producción de biodiesel. Revista Tecnología y Ciencia, Universidad Tecnológica Nacional 26:87-92.

12. Morales W. G.; Polich N.; Sequeira A.; Chamorro E.; Herrero E.; (2010).Purificación de glicerol obtenido a partir de síntesis de biodiesel de aceite crudo de algodón. Avances en Energías Renovables y Medio Ambiente. 14 (6):25-30.

13. Sequeira; A.; Polich; N.; Morales; W. y Chamorro; E.; (2011). Primeros pasos en la obtención de biodiesel utilizando un reactor continuo de construcción sencilla. AVERMA; Editorial INENCO; Avances en Energías Renovables y Medio Ambiente. 15 (9):47-55.

14. Morales W. G.; Dagnino P.; Diaz A.; Polich N.; Sequeira A. y Chamorro E.; (2009). Proceso semicontínuo en la transesterificación de triglicéridos de semilla de algodón. ISBN 978-987-575-079-1. CLICAP.

15. Morales W. G.; Dagnino P.; Díaz A.; Sequeira A.; Chamorro E. y Herrero E.; (2009). Transesterificación de trigliceridos de algodón catalizada por alcalis. AVERMA. 13 (6):87-92.

16. Naik S .N.; Goud V. V.; Rout P. K. y Dalaib A. K.; (2010). Production of first and second generation biofuels: A comprehensive review. Renewable and Sustainable Energy Reviews. 14 (2):578-597.

17. Nigama P. S.; y Singh A.; (2011). Production of liquid biofuels from renewable resources Progress in Energy and Combustion Science. Volume 37 (1):52-68.

18. Xue J.; Grift T. E. y Hansen A. C.; (2011). Effect of biodiesel on engine performances and emissions. Renewable and Sustainable Energy Reviews Volume 15:1098-1116. 
19. Morales W.; Dagnino P.; Díaz A.; Sequeira A. Y Chamorro E.; (2008). Tratamiento de aceite crudo de algodón para transesterificación alcalina. Avances en Energías Renovables y Medio Ambiente. 12 (6):9598.

20. Morales W.G.; Dagnino E.P.; Díaz A.; Chamorro E; Herrero E.; (2009). Estudio cinético de la metanólisis de triglicéridos de aceite de algodón; Tercer Congreso Nacional - Segundo Congreso Iberoamericáno Hidrógeno y Fuentes Sustentables de Energía - HYFUSEN.

21. Yusuke Asakuma; Kouji Maeda; Hidetoshi Kuramochi; Keiseuke Fukui;(2009).Theoretical study of the transesterification of triglycerides to biodiesel fuel; Fuel 88:786-791.

22. Huppes da Silva Augusto Cesar, Kuhnen Carlos Alberto, da Silva Sebastião Claudino, Dall'Oglio Evandro L., de Sousa Paulo T. Jr.; (2013). DFT study of alkaline-catalyzed methanolysis of pentylic acid triglyceride: Gas phase and solvent effects. Fuel 107:387-393.

23. Sequeira, Alfredo; Chamorro, Ester; Zalazar, Maria F.; Acuña, Victor y Peruchena, Nélida M.; (2006) Propiedades atomicas integradas de la densidad de carga electronica en analogos de feromonas. En Actas del XXVI Congreso Argentino de Química, Setiembre, San Luis, Argentina. ISBN-10: 987-1031-45-9.

24. Chamorro, E., Benitez, E., Sequeira, A. and Peruchena, N. M.; (2008) Conformational and electronic study of sex pheromone of the pine processionary moth and some related derivative compounds with modification in the polar group. J. Argent. Chem. Soc. 96(1-2):62-79.

25. Chamorro, E., Sequeira, A., Zalazar M. F. and Peruchena, N. M.; (2008) Theoretical analysis of the electronic properties of the sex pheromone and its analogue derivatives in the female processionary moth thaumetopoea pytiocampa. Bioorganic \& Medicinal Chemistry 16(18):8535-8545.

26. Alfredo F. Sequeira, Liliana M. Caceres, Cristian M. Gauna, Walter G. Morales, Ester R. Chamorro (2017). Revista Tecnología y Ciencia, Universidad Tecnológica Nacional. 30:169-177. Modelado matemático de la cinética de la reacción de transesterificación y prospección probabilística de la composición de lípidos saponificables en aceites vegetales. 